\title{
STATISTICALLY OPTIMAL INTERSLICE VALUE INTERPOLATION IN 3D MEDICAL IMAGING: THEORY AND IMPLEMENTATION
}

\author{
RoB W. PARROTT, MARTIN R. STYTZ ', PHIL AMBURN, DAVID ROBINSON \\ AIR FORCE INSTITUTE OF TECHNOLOGY \\ WRIGHT-PATTERSON AFB, OH 45433
}

\section{ABSTRACT}

We describe a technique for statistically optimal interslice interpolation of scalar values for use in threedimensional medical image rendering. The interpolation technique is based upon kriging. Kriging is known to be the best linear unbiased estimation technique for spatially distributed data. This paper presents the results obtained using kriging in the object space pre-processing operation of slice interpolation by slice-value interpolation. As a byproduct of the technique, kriging calculates the estimation error for the interslice values. This allows us to quantify the interpolation error in slices computed by the estimation technique.

\section{INTRODUCTION}

Three-dimensional (3D) medical imaging algorithms estimate values within the $3 \mathrm{D}$ scalar field of data to enhance the image or to create a uniformly sampled scene. This estimation can be performed in the object space pre-processing operation of slice interpolation ([16], [17]). Commonly used deterministic interpolation techniques, such as nearest neighbor, linear, trilinear, and tricubic interpolation (as described in [18]), can inaccurately estimate these values. As a result, unquantified error is introduced into the rendered image. Our research has been aimed at both reducing and quantifying this error.

In this paper we describe a technique for statistically optimal interslice interpolation. The interpolation technique is based upon the geo-statistical process called kriging. Kriging is known to be the best linear unbiased estimation technique for spatially distributed data ([1], [5], [6], and 19]). Because of this property of kriging, we theorized that it could be used to interpolate medical imaging modality data. We also expected that the images produced with kriging would have minimal error and would appear noticeably better than images produced with other interpolation techniques. As a byproduct of the technique, kriging calculates the estimation error for the interslice values. This error value allows us to quantify the interpolation error in each slice.

The next section presents a background on kriging. The third section describes our implementation of kriging. The fourth section of the paper contains the results of using kriging and iwo deterministic methods, linear and tricubic interpolation, for estimating interslice values by slice-value interpolation. The last section of the paper presents our conclusions and recommendations for further work.

\section{KRIGING BACKGROUND}

Kriging is a statistical process that estimates a value at a point using the spatially dependent values in a neighborhood or zone near the point. Kriging was originally developed to estimate ore reserves and thicknesses or accumulations in mining deposits, [12], but is applicable to any spatially dependent data. The kriging process is based upon the concept of using regionalized variables to model spatially dependent data. Kriging makes the common assumption that there is a spatial, linear relationship between the unknown value at a point and the known values of neighboring points. Kriging computes the value for the unknown data point using a weighted linear sum of known data values. The known data values represent instances of regionalized variables. The weights are chosen to minimize the estimation error variance and provide unbiasedness in the sampling, making kriging the best linear unbiased estimate for the data. The process is termed best because the weights are chosen to minimize the estimation error variance and to provide unbiasedness in the sampling. As a byproduct of the kriging process, an estimate of the error variance for each interpolated point value is produced, allowing us to quantify the error at each point in an interpolant slice and in the interpolant slice itself.

\footnotetext{
$\dagger_{\text {Address correspondence to this author. }}$
} 
Kriging departs from other techniques for scalar value interpolation in two regards. First, it bases its estimates upon a large neighborhood of points and treats those points as regionalized variables instead of random variables. The use of regionalized variables recognizes the existence of regions or zones of influence in the data. In kriging, each region is analyzed to determine correlation or inter-dependence among the data in the region. Second, kriging computes both a local and a global statistical characterization of the data set, allowing it to account for drift and other anomalies in the data that can cause inaccuracies in the interpolated estimate of the value at a point. Additional details and background on kriging theory are presented in [1], [3], [4], [5], [6], [7], [8], [9], [12], [13], [14], and [15].

Kriging is a modified linear regression technique that calculates a weighted linear sum of sample values to produce its estimates. There are two broad classes of kriging, ordinary kriging and universal kriging. In ordinary kriging, the mean value for the data in a region is unknown and the mean is assumed to be stationary throughout the region. In universal kriging, the mean is again unknown, but the mean is not stationary throughout the region. Sample means that differ from place to place are called drifts (also trends or non-stationary means). Drift within the local neighborhood of sample values is a location dependent drift, as discussed in [9]. Global drifts are averages computed from a region of sample values that can extend beyond the support. Global drift is dealt with differently, see [5]. We discuss ordinary kriging first and then describe universal kriging.

Equation (1) is the kriging equation. In that equation the goal is to estimate $\hat{z}$, the unknown value at the position $p$ within the neighborhood of known points $p_{i}$ and known values $z_{i}\left(p_{i}\right)$. $z$ is the actual value at point $p$.

$$
\hat{z}(p)=\sum_{i=1}^{n} w_{i} z_{i}\left(p_{i}\right)
$$

The $z_{i}$ s are the regionalized variables with the parameter being an $n$-dimensional point and the $w_{i}$ s the weights. Optimal weights are determined by enforcing the following two conditions. These conditions make $\hat{Z}$ the best linear unbiased estimator:

$$
\begin{aligned}
& \mathcal{E}(\hat{Z}-Z)=0 \\
& \mathcal{E}(\hat{Z}-Z)^{2} \text { is minimum }
\end{aligned}
$$

The estimation error, $\hat{z}-z$, is a measure of the dissimilarity between the two variables $\hat{z}$ and $z$. $\mathcal{E}(\hat{z}$ $z)^{2}$ is the mean square error and $\mathcal{E}$ is the expected value or mean.

These two conditions are the base from which the kriging system of equations, used to find the $w_{i}$ 's, are derived. The constraints, and hence the system, change depending on the behavior of the sample means. For the moment, assume the sample means are unknown and no drifts exists in the data. In ordinary kriging the unbias constraint forces the weights to sum to 1 because the mean, $m(p)$, is constant, producing equation (5). These assumptions lead to the following system of equations for ordinary kriging.

$$
\begin{aligned}
& \sum_{j} w_{i} \gamma\left(f_{i j}\right)+\eta=\gamma\left(f_{i p}\right) \\
& \sum_{j} w_{i}=1
\end{aligned} \quad(i=1, \ldots, n)
$$

Here, $\gamma\left(f_{i j}\right)$ is the semivariogram approximating the covariance between sample points $i$ and $j$ a distance of $f$ apart. The semivariogram is a function that measures the degree of spatial dependence between sample values along a specific orientation. The semivariogram is discussed in a later section. Solving the ordinary system of equations will yield optimal weights for the kriging equation, equation (1) above.

Universal kriging relaxes the assumption that drift does not exist in the data. The kriging system of equations change because the presence of drift forces the unbias constraint to change. David [5] expresses the drift at a point $p$ as: 


$$
m(p)=\mathcal{E}(Z(p))=\sum_{\ell=0}^{k} d_{l} f^{l}(p)
$$

Equation (6) is normally implemented as a finite order polynomial. The $f^{f}(p)$ 's are $k+1$ known functions, usually monomials, and the $d f$ 's are the $\mathrm{k}+1$ unknown drift coefficients. Now that $m(p)$ is no longer constant but takes into account drift, the unbias constraint results in a new set of equations:

$$
\begin{aligned}
& \mathcal{E}(\hat{z}-z)=0 \\
& \mathcal{E}\left(\sum_{i} z\left(p_{i}\right)\right)-\sum_{k} d_{f} f^{f}(p)=0 \\
& \sum_{i} w_{i} f^{f}\left(p_{i}\right)=f^{f}(p) \quad(\mathcal{L}=0,1, \ldots, k)
\end{aligned}
$$

Notice that the drift coefficients, $d_{l}$, have dropped out of the constraint in equation (7). Thus the universal system is independent of the drift coefficients, but still insures unbiasedness. Since this condition insures unbiasedness regardless of the unknown drift coefficients $d_{f}$, the term universal is used to denote the system of equations that result, as described in [9]. This constraint, equation (7), adds $k+1$ more equations to the minimum variance condition, thus $k+1$ additional Lagrange Multipliers, $\eta$, are needed $([2],[7])$. After the partial derivatives of the equations are taken with respect to the $n$ weights and the $k+1$ Lagrangian Multipliers and set to zero, the universal kriging system of equations is obtained.

$$
\begin{array}{ll}
\sum_{j=1}^{n} w_{i} \gamma_{i j}+\sum_{i=0}^{\ell} \boldsymbol{\eta}_{l} f^{l}\left(p_{i}\right)=\gamma_{i p} & (i=1, \ldots, n) \\
\sum_{i=1}^{n} w_{i} f^{l}\left(p_{i}\right)=f^{l}(p) & (1=0,1, \ldots, k)
\end{array}
$$

In (8), the requirement for minimum estimation variance is met, and (9) describes the non-biasedness constraint. Solving this system of equations yields the optimal weights for the kriging equation, (1).

Before either the universal or the ordinary kriging system of equations can be solved, a model semivariogram must be determined to account for the interdependencies of the data. The semivariogram is used to approximate the covariance between sample points. The semivariogram also indicates the spatial dependence of regionalized variable instances (the known sample values). The theoretical semivariogram is defined as:

$$
2 \gamma(h)=\operatorname{var}\left(Z\left(p_{i}+f\right)-Z\left(p_{i}\right)\right) \quad \forall x_{i} \quad x_{i}+\hbar \in \mathcal{N}
$$

where $f$ is a distance. Thus, the semivariance only depends on the two variable's relative positions in space. The two types of semivariograms used in kriging are the experimental and model semivariograms. The experimental semivariogram is keyed directly to the data at hand and so produces an accurate description of the covariance. However, the experimental semivariogram only computes function values for a fixed value of $f$ and this typically does not coincide with the spacing of the sample data. As a result, the experimental semivariogram can't be used directly to krige the data. To krige the data, a model semivariogram is used. Model semivariograms are generic templates that are used to describe broad classes of spatial dependence in data. A model semivariogram allows any distance, $f$, as a function parameter. Once the experimental semivariogram is calculated, its graph is compared to known model semivariograms. When a match is found, suitable parameters for the model semivariogram are determined. The model supplies the $\gamma\left(f_{i j}\right)$ needed to krige the data.

\section{IMPLEMENTATION}

To interpolate medical imaging modality slice data, we calculate the values along the edge of a 
computational cell. A computational cell is a parallelepiped. Four vertices of the parallelpiped lie in one dat a slice and the other four lie in a single adjacent slice, as shown in Figure 1.

The slice interpolation algorithm has three basic steps. First, read in four medical data slices. Four slices
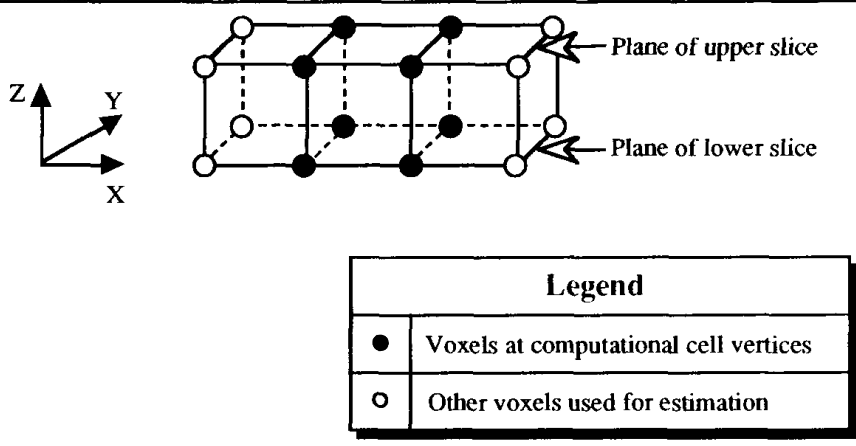

Figure 1: Computational cell in a neighborhood of 16 voxel values.

are needed because tricubic interpolation needs 64 sample control values. When estimating, 16 values come from each of the two image planes the cell lies between (as shown in Figure 1), and 16 values come from each of the planes immediately adjacent to these. Second, march the cell (cube) between the two inner data slices (as in "marching cubes", [121). Third, estimate values for new slice(s) along cell edges.

One of three estimation techniques, either linear interpolation, tricubic interpolation, or kriging, is applied in step three. Our implementation of linear interpolation is standard. The technique we used for tricubic interpolation is similar to the one described in [18]. The kriging implementation is discussed next.

Our implementation makes some assumptions about the data that make the kriging of medical imaging data practical.. We assume that the data is isotropic and that the model semivariogram is known, allowing us to forego the semivariogram analysis phase of kriging. We also assume that only local drift is present. We experimented with both quadratic and linear local drift, models and determined that the difference in the results was insignificant for the data. Therefore, we present only the computationally more efficient linear drift results. The three-dimensional linear drift expression in terms of geometric coordinates $\left(x_{i}, y_{i}, z_{i}\right)$ is:

$$
m\left(p_{i}\right)=m\left(x_{i}, y_{i}, z_{i}\right)=d_{0}+d_{1} x_{i}+d_{2} y_{i}+d_{3} z_{i}
$$

This equation points out the location dependence of the drift. Our assumed model semivariogram, or drift model, is a cubic function ([10]). That is:

$$
\gamma(h)=a b s\left(f^{3}\right)
$$

This function was chosen to model the variation assumed by the tricubic function. We also experimented with $h$ and $\hbar^{2}$ model semivariograms. In our experience, the assumed model does not greally affect the accuracy of the estimation. The system of universal kriging equations is represented in matrix form as $\mathbf{A X}=\mathbf{B}$, where the $\mathbf{X}$ column vector contains the kriging weights. We tested kriging using neighborthood sizes of $8,16,32$, and 64 voxels. A neighborhood size of 8 is just the computational cell. The orientation and sampling geometry for 16 and 32 voxel neighborhoods are depicted in Figure 2.

\section{RESULTS}

We present the results of two experiments that compare kriging with linear and tricubic interpolation. The photographs contain 18 images depicting 2D medical image slices. The top four images are the original contiguous slices. The 14 remaining images are estimated slices that lie between the two middle images in the top row. These 14 images were generated using different interpolation techniques. The second row from the top has two images, one computed by linear interpolation, the other by tricubic interpolation. All the images in the last two rows were derived using different forms of kriging. The image titles describe the operations and assumptions used to compute the image. For example, the image titled krigenh8linear was 
computed with kriging using a neighborhood size of 8 voxels and assuming local linear drift.

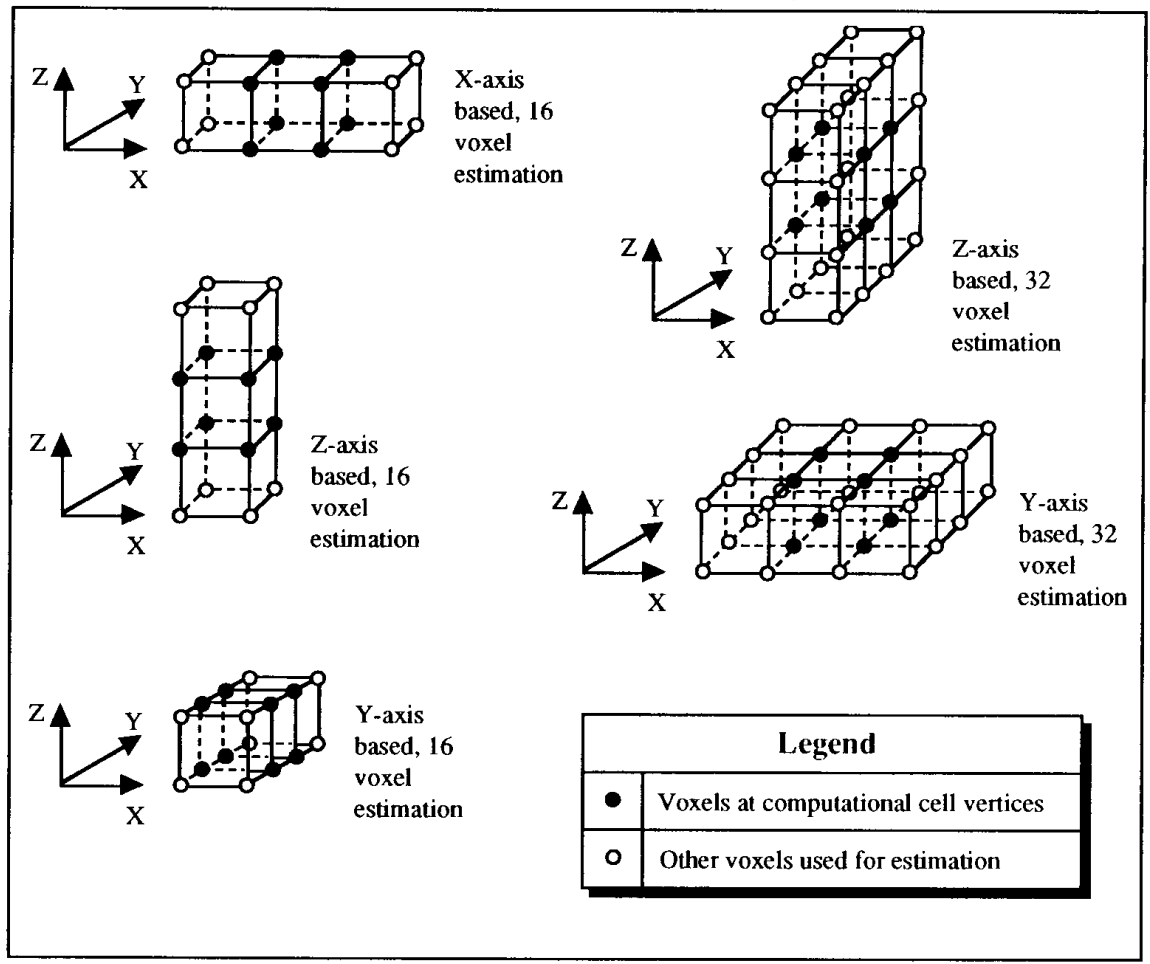

Figure 2: Orientation and geometry of 16 and 32 voxel neighborhoods used in kriging estimation.

In Tables 1 and 2, the entries in the Image and Image Compared With columns refer to image titles. The last column in each table indicates the percentage of the average difference in the gray scale range (0-255). The first study uses 202-pixel x 132-pixel CT slices of a dog's heart that have a $1 \mathrm{~mm}$ inter-slice distance. Figure 3 presents the photographic results for this study. The goal of this study was to estimate the values in a new slice that lies between original data slices 41 and 42 . Table 1 presents results pertaining to these images. We observed that the image generated by tricubic interpolation was inaccurate. Also, the images produced by kriging with a neighborhood 64 voxels are inaccurate and its values are similar to the tricubic interpolation. Except for kriging with neighborhood of $16^{\prime} \mathrm{Z}$ ', kriging with smaller neighborhoods estimates values closer to trilinear than to tricubic, and most closely approximates trilinear with a neighborhood of 8 voxels, assuming local linear drift. Ordinary kriging with smaller neighborhood sizes of 16 voxels and 8 voxels also produces errors in the estimated values. Universal kriging (local drift assumed) corrects these errors and is the best choice for interpolating function.

The second study uses 166-pixel x 166-pixel MRI slices of a three month old baby's head. In this case, the interslice spacing is $4 \mathrm{~mm}$. The goal for this study was to estimate the values in a new slice that lies between original data slices 31 and 32 . The results for this study are shown in Figure 4 and Table 2 . Again, universal kriging is the best choice for the interpolating function.

The images produced using kriging are, in our opinion, visually comparable to images produced using trilinear and tricubic interpolation. While the images for the second study generally look similar, the values in Table 2 show that the estimations in this study are similar to those in the dog heart study, presented in Table 1 .

The erroneous images produced by tricubic interpolation and several of the kriging variations could be 
caused by invalid assumptions about the data variation. By using a smaller neighborhood size for kriging, we modified our assumptions about data variation and our results show that better images were obtained.

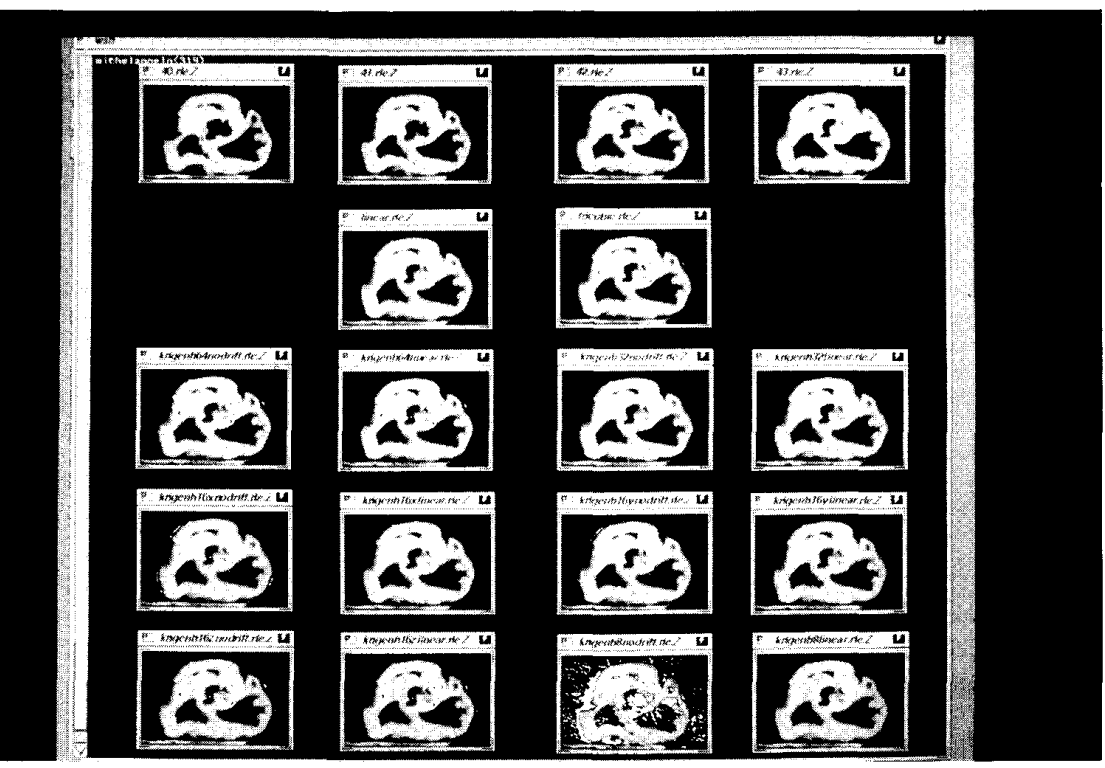

Figure 3: Slices computed from CT data. Interpolation techniques were used to create a new slice between original slices 32 and 33 . Window titles depict the type of interpolation used.

\begin{tabular}{|l|l|c|c|c|}
\hline \multicolumn{5}{|c|}{ Table 1: Comparison of Dog Heart CT Estimated Values. } \\
\hline \multicolumn{1}{|c|}{ Image } & \multicolumn{1}{|c|}{$\begin{array}{c}\text { Largest } \\
\text { With }\end{array}$} & $\begin{array}{c}\text { Average } \\
\text { Difference of } \\
\text { Values }\end{array}$ & $\begin{array}{c}\text { Percentage } \\
\text { Difference }\end{array}$ \\
\hline \hline krigenh64nodrift & kimated \\
krigenh64linear & tricubic & 0.6229 & 0.0196 & 0.0077 \\
krigenh64linear & linear & 4.1242 & 0.1365 & 0.0533 \\
\hline krigenh32nodrift & krigenh32linear & 18.0780 & 0.6781 & 0.2649 \\
krigenh32linear & tricubic & 0.9815 & 0.0416 & 0.0163 \\
krigenh32linear & linear & 12.1747 & 0.5519 & 0.2156 \\
\hline krigenh16xnodrift & krigenh16xlinear & 3.2616 & 0.1295 & 0.0506 \\
krigenh16xlinear & tricubic & 30.0089 & 1.3080 & 0.5109 \\
krigenh16xlinear & linear & 12.4780 & 0.5407 & 0.2112 \\
\hline krigenh16ynodrift & krigenh16ylinear & 02.2749 & 0.0785 & 0.0307 \\
krigenh 16ylinear & tricubic & 70.8097 & 0.9918 & 0.3870 \\
krigenh16ylinear & linear & 12.7687 & 0.5527 & 0.2159 \\
\hline krigenh16znodrift & krigenh16zlinear & 3.0524 & 0.1129 & 0.0441 \\
krigenh16zlinear & tricubic & 24.5295 & 0.4502 & 0.1759 \\
krigenh16zlinear & linear & 2.7525 & 0.1125 & 0.0439 \\
\hline krigenh8nodrift & krigenh8linear & 17.1736 & 0.6742 & 0.2634 \\
krigenh8linear & tricubic & 203.1685 & 10.8014 & 4.2190 \\
krigenh8linear & linear & 14.4007 & 0.5639 & 0.2203 \\
\hline
\end{tabular}

Another cause of error might be anisotropy in the data. We believe that the data are anisotropic in the ' $Z$ ' direction (relative to ' $\mathrm{X}$ and ' $\mathrm{Y}$ '). We believe that ' $\mathrm{Z}$ ' direction anisotropy exists because kriging using a 
neighborhood of $16^{\prime} \mathrm{Z}$ ' produces gross inaccuracies like tricubic (see krigenh16zlinear results), whereas neighborhoods of $16^{\prime} \mathrm{Y}$ and $16^{\prime} \mathrm{X}$ ' do not. The inaccuracies corrected by assuming local linear drift

\begin{tabular}{||l|l|c|c|c|}
\hline \multicolumn{5}{|c|}{ Table 2: Comparison of Baby Head MRI Estimated Values. } \\
\hline \multicolumn{1}{|c|}{ Image } & \multicolumn{1}{|c|}{$\begin{array}{c}\text { Image Compared } \\
\text { With }\end{array}$} & $\begin{array}{c}\text { Largest } \\
\text { Estimated } \\
\text { Difference }\end{array}$ & $\begin{array}{c}\text { Average } \\
\text { Difference of } \\
\text { Values }\end{array}$ & $\begin{array}{c}\text { Percentage } \\
\text { Difference }\end{array}$ \\
\hline \hline krigenh64nodrift & \multicolumn{1}{|c|}{ krigenh64linear } & 1.8551 & 0.0571 & 0.0223 \\
krigenh64linear & tricubic & 6.7329 & 0.3772 & 0.1473 \\
krigenh64linear & linear & 23.0072 & 1.4960 & 0.5844 \\
\hline krigenh32nodrift & krigenh32linear & 3.4845 & .09 .32 & 0.0364 \\
krigenh32linear & tricubic & 17.1510 & 1.0481 & 0.4094 \\
krigenh32linear & linear & 5.1335 & 0.2807 & 0.1097 \\
\hline krigenh16xnodrift & krigenh16xlinear & 96.5233 & 3.4776 & 1.3580 \\
krigenh16xlinear & tricubic & 17.3192 & 1.0851 & 0.4239 \\
krigenh16xlinear & linear & 4.2738 & 0.2252 & 0.0880 \\
\hline krigenh16ynodrift & krigenh16ylinear & 70.1153 & 2.5202 & 0.9845 \\
krigenh 16ylinear & tricubic & 17.4163 & 1.0853 & 0.4239 \\
krigenh 16ylinear & linear & 5.2594 & 0.2249 & 0.0878 \\
\hline krigenh16znodrift & krigenh16zlinear & 21.8722 & 1.0342 & 0.4040 \\
krigenh 16zlinear & tricubic & 4.1201 & 0.2519 & 0.0984 \\
krigenh16zlinear & linear & 21.4664 & 1.4106 & 0.5510 \\
\hline krigenh8nodrift & krigenh8linear & 227.1227 & 16.3677 & 6.3940 \\
krigenh8linear & tricubic & 17.9036 & 1.1631 & 0.4543 \\
krigenh8linear & linear & 0.7859 & 0.0282 & 0.0110 \\
\hline
\end{tabular}

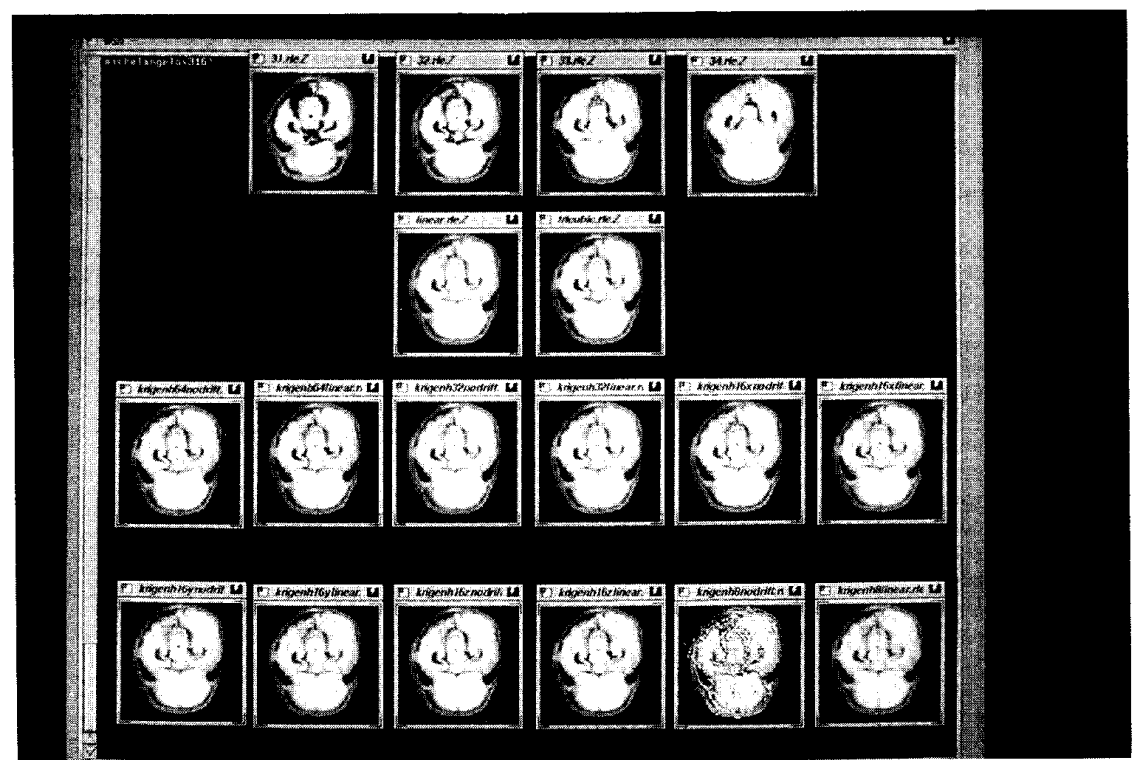

Figure 4: Slices computed from MRI data. Interpolation techniques were used to create a new slice between original slices 32 and 33. Window titles depict the type of interpolation used.

indicate that the sample means are locally non-stationary. Our results also indicate that local drift assumptions have a greater effect on estimation accuracy than the assumed model semivariogram. 


\section{CONCLUSIONS AND FUTURE WORK}

We have shown that kriging produces images that are visually comparable or better than images produced using trilinear and tricubic interpolation. Kriging has a twofold appeal. First, the technique can quantify the interpolation error, via the estimation variance, in the rendered image. Second, because the interpolation error can be made low, there is a high degree of confidence associated with the interpolated values and the rendered images' accuracy.

Our current work is centered around decreasing the time required to generate images. Our current implementation is slow. The time to generate one 2D slice using a neighborhood of 16 voxels is approximately an hour. One acceleration technique we are exploring is to dynamically determine when to invert the A matrix. In the current implementation, the $\mathbf{A}$ matrix is inverted for every new value estimated. However, since portions of the A matrix never change, it is probable not necessary to invert it for every value. Once the A matrix inversion issue is resolved, we intend to accelerate performance further by parallelizing the implementation. We plan to restructure the code so that the $3 \mathrm{D}$ data are partitioned among multiple processors. This data-parallel implementation would minimize inter-process communication since each portion of the data could be kriged independently. We are also exploring the use of kriging for volume visualization.

\section{REFERENCES}

[1] Clark, Isobel. Practical Geostatistics, Applied Science Publishers Ltd: London, 1979.

[2] Cressie, Noel. "Kriging Nonstationary Data", Journal of the American Statistical Association, vol. 81 , no. 395, pp. 625-634, Sep. 1986.

[3] Cressie, Noel. "Geostatistics", The American Statistician, vol. 43, no. 4, pp. 197-202, Nov. 1989.

[4] Cressie, Noel. "The Origins of Kriging", Mathematical Geology, vol. 22, no. 3, pp. 239-52, 1990.

[5] David, Michael. Geostatistical Ore Reserve Estimation, Elsevier Scientific Publishing Company: New York, New York, 1977.

[6] Davis, John C. Statistics and Data Analysis in Geology, 2nd Edition, John Wesley \& Sons: New York, New York, 1986

[7] Dubrule, Olivier. "Comparing Splines and Kriging", Computers \& Geosciences, vol. 10, no. 2-3, pp. 327-38, 1984.

[8] Journel, A. G. and Huijbregts, Ch. J. Mining Geostatistics, Academic Press: New York, New York, 1978.

[9] Journel, Andre G. Fundamentals of Geostatistics in Five Lessons, American Geophysical Union: Washington, D. C., 1989.

[10] Kerbs, Lynda. "GEO-Statistics: The Variogram", COGS Computer Contributions, vol. 12, no. 2, pp. 54-59, Aug. 1986.

[11] Lorensen, William E. and Cline, Harvey E. "Marching Cubes: A High Resolution 3D Surface Construction Algorithm," Computer Graphics, vol. 21, no. 4, pp. 163-169, July 1987.

[12] Matheron, G. "Principles of Geostatistics", Economic Geology, vol. 58, pp. 1246-1266, 1963.

[13] Matheron, G. "Principles of Geostatistics", in Statistical Analysis in Geology, Cubbit, John M. and Henley, Stephen (eds), Dowden, Hutchinson, \& Ross, Inc.: Stroudsburg, 1978.

[14] Matheron, G. "Splines and Kriging: Their Formal Equivalence", Proceedings of Down to Earth Statistics: Solutions Looking for Geological Problems. Syracuse University, Geology Contribution 8, pp. 77-95, 1981.

[15] Oliver, M. A. and Webster, R. "Kriging: A Method of Interpolation for Geographical Information Systems", International Journal of Geographical Information Systems (UK), vol. 4, no. 3, pp. 313 32, July-September, 1990.

[16] Stytz, Martin R., Frieder, Gideon; and Frieder, Ophir. "Three-Dimensional Medical Imaging: Algorithms and Computer Systems," ACM Computing Surveys, vol. 23, no. 4, pp. 421-500, Dec. 1991.

[17] Udupa, Jayaram K. and Herman, Gabor T. 3D Imaging in Medicine, CRC Press: Boston, 1991.

[18] Wilhelms, Jane and Van Gelder, Allen. "Topological Considerations in Isosurface Generation", Computer Graphics, vol. 24, no. 5, pp. 79-86, November, 1990. 